NIST Handbook 150-8

\title{
NVLAP \\ Acoustical Testing Services
}

Kari K. Harper

This publication is available free of charge from:

https://doi.org/10.6028/NIST.HB.150-8-2018

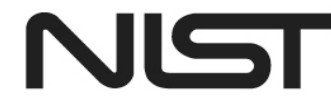

National Institute of Standards and Technology U.S. Department of Commerce 


\title{
NIST Handbook 150-8
}

\section{NVLAP \\ Acoustical Testing Services}

\author{
Kari K. Harper \\ National Voluntary Laboratory Accreditation Program \\ Standards Coordination Office \\ Laboratory Programs
}

This publication is available free of charge from:

https://doi.org/10.6028/NIST.HB.150-8-2018

November 2018

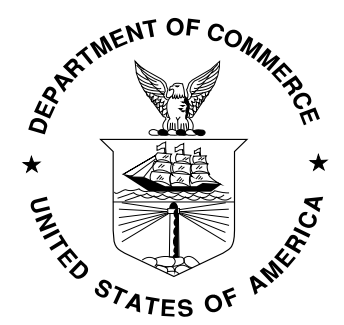

U.S. Department of Commerce Wilbur L. Ross, Jr., Secretary

National Institute of Standards and Technology Walter Copan, NIST Director and Undersecretary of Commerce for Standards and Technology 


\section{NVLAP AND THE NVLAP LOGO}

The term NVLAP and the NVLAP logo are federally registered certification marks of the National Institute of Standards and Technology and the federal government, who retain exclusive rights to control the use thereof. Permission to use the term and/or logo is granted to NVLAP-accredited laboratories for the limited purposes of announcing their accredited status, and for use on reports that describe only testing and calibration within the scope of accreditation. NIST reserves the right to control the quality of the use of the term NVLAP and of the NVLAP logo. 


\section{Foreword}

The NIST Handbook 150 publication series sets forth the procedures, requirements, and guidance for the accreditation of testing and calibration laboratories by the National Voluntary Laboratory Accreditation Program (NVLAP). The series is comprised of the following publications:

- $\quad$ NIST Handbook 150, NVLAP Procedures and General Requirements, which contains the general procedures and requirements under which NVLAP operates as an unbiased third-party accreditation body;

- $\quad$ NIST Handbook 150-xx program-specific handbooks, which supplement NIST Handbook 150 by providing additional requirements, guidance, and interpretive information applicable to specific Laboratory Accreditation Programs (LAPs) under NVLAP.

The program-specific handbooks are not stand-alone documents, but rather are companion documents to NIST Handbook 150. Each program-specific handbook tailors the general criteria found in NIST Handbook 150 to the specific test methods, calibrations, or types of tests or calibrations covered by a LAP.

NIST Handbook 150-8, NVLAP Acoustical Testing Services, presents the technical requirements and guidance for the accreditation of laboratories under the NVLAP Acoustical Testing Services LAP. The 2018 edition of NIST Handbook 150-8 supersedes and replaces the 2011 edition.

The handbook was revised with the participation of technical experts in the field of acoustical testing services and was approved by NVLAP. The following significant changes have been made to this handbook with respect to the previous edition:

- the numbering has been updated to reflect that used by ISO/IEC 17025:2017, General requirements for the competence of testing and calibration laboratories; (hereafter referred to as ISO/IEC 17025)

- requirements that closely duplicated existing requirements in either ISO/IEC 17025 or NIST Handbook 150 have been removed.

- $\quad$ wording changes for clarification have been made where needed.

This handbook is also available on the NVLAP website (http://www.nist.gov/nvlap) under NVLAP Publications and Forms.

Questions or comments concerning this handbook should be submitted to NVLAP, National Institute of Standards and Technology, 100 Bureau Drive, Stop 2140, Gaithersburg, MD, 20899-2140; phone: 301-975-4016; fax: 301-926-2884; e-mail: nvlap@nist.gov. 


\section{Introduction}

In 1980, the Department of Commerce (DOC) received a formal request from Owens-Corning Fiberglas Corporation that the need for accreditation of acoustical testing laboratories be determined. After gathering information, the DOC published a final finding of need to accredit laboratories that provide acoustical testing services (46 FR 51267-512271). Public hearings and workshops were held, and the laboratory accreditation program for Acoustical Testing Services was formally established by the National Bureau of Standards in 1982 (47 FR 39874-39878). NVLAP accreditation is based on the evaluation of a laboratory's technical qualifications and competence for conducting specific standard test methods in acoustical testing.

Test methods that relate to this program may be developed by a trade, professional, governmental, or standards-writing organization.

Accreditation is available to any laboratory (including commercial, manufacturer, university, and federal, state and local government laboratories) that tests in accordance with standard acoustical test methods. A foreign-based laboratory may also be accredited by NVLAP if the laboratory meets the same requirements as domestic laboratories and pays any required additional fees associated with conducting the on-site assessment.

To be granted accreditation, a laboratory shall satisfy the NVLAP requirements contained in ISO/IEC 17025, NIST Handbook 150 and this handbook, and in the checklists for NIST Handbook 150 and this handbook. 


\begin{abstract}
NIST Handbook 150-8 presents technical requirements and guidance for the accreditation of laboratories under the National Voluntary Laboratory Accreditation Program (NVLAP) Acoustical Testing Services program. It is intended for information and use by accredited laboratories, laboratories seeking accreditation, laboratory accreditation systems, users of laboratory services, and others needing information on the requirements for accreditation under this program. The 2018 edition of NIST Handbook 150-8 incorporates changes resulting from the release of the newest editions of ISO/IEC 17025, General requirements for the competence of testing and calibration laboratories, and NIST Handbook 150, NVLAP Procedures and General Requirements, as well as editorial improvements. The requirements of NIST Handbook 150, ISO/IEC 17025, and the specific requirements in NIST Handbook 150-8, must be combined to produce the criteria for accreditation in the NVLAP Acoustical Testing Services program.
\end{abstract}

\title{
Keywords
}

Accreditation; acoustical testing; conformity assessment; laboratory; management system; NVLAP; on-site assessment; testing. 


\section{Contents}

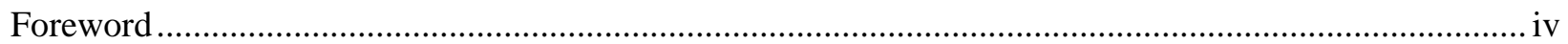

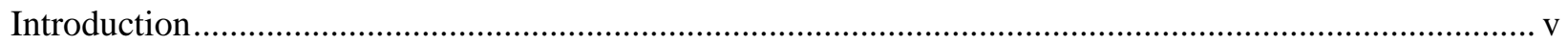

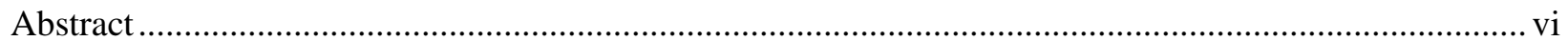

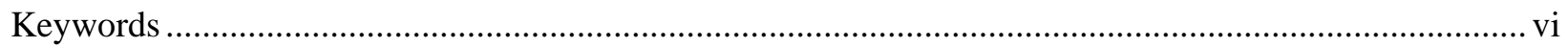

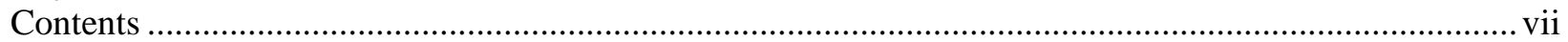



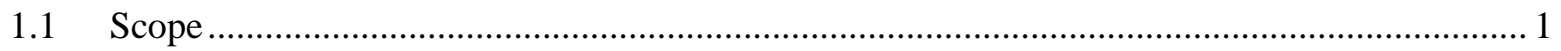

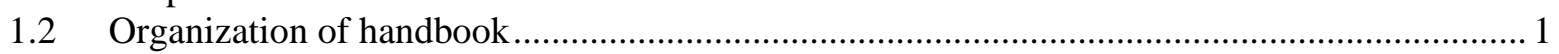

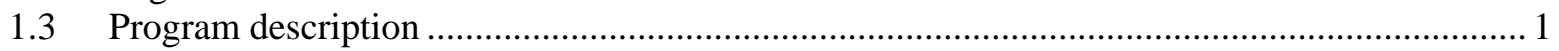

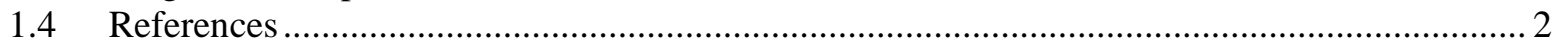

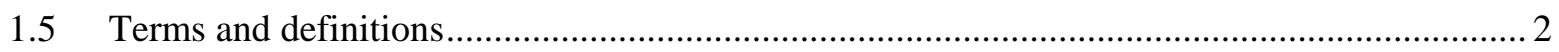

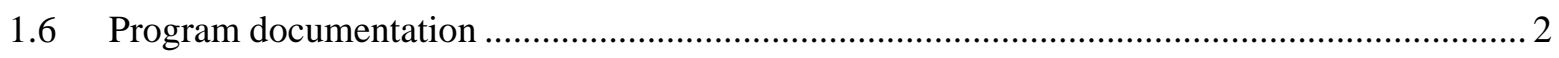

2 LAP establishment, development and implementation ................................................................ 3

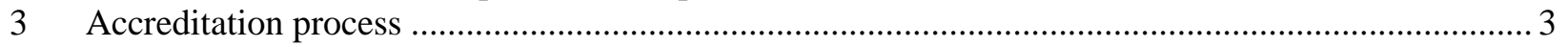

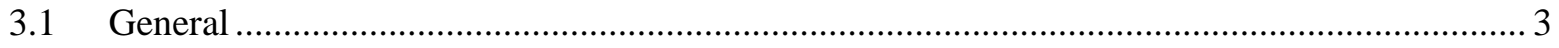

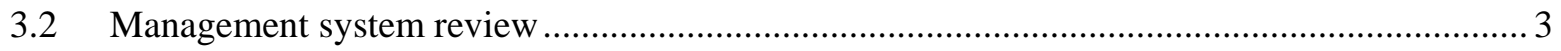

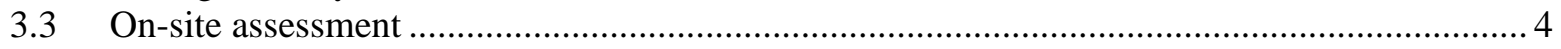

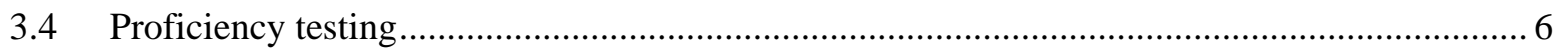

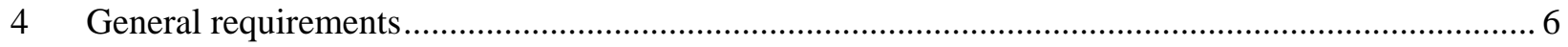

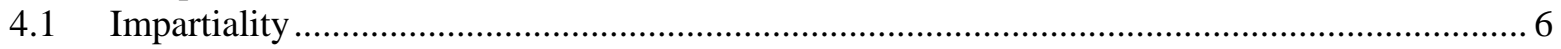

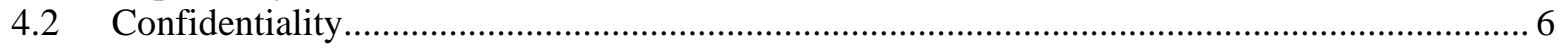

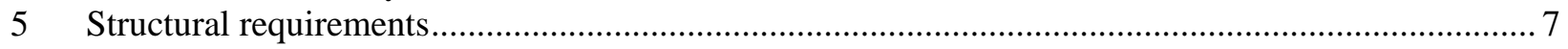

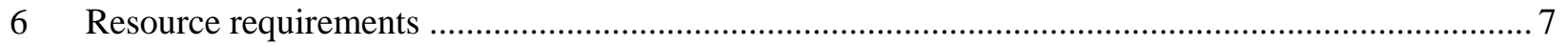

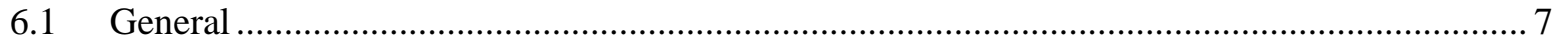

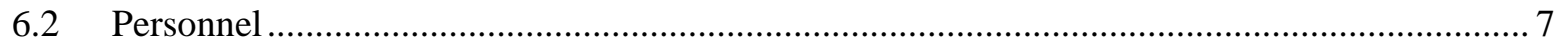

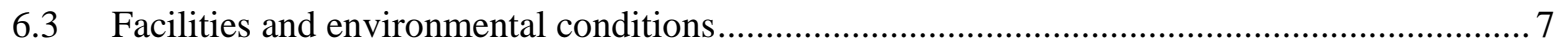

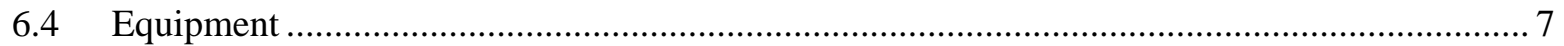

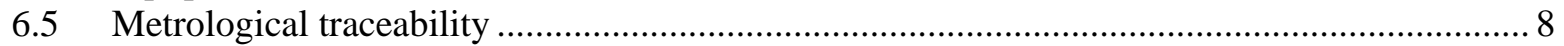

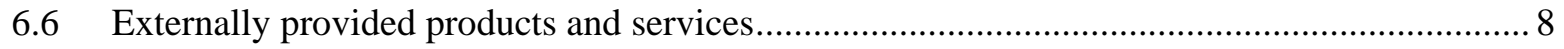

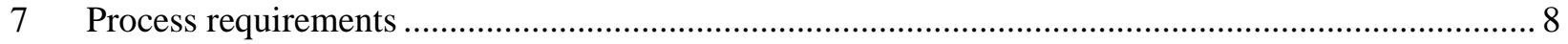

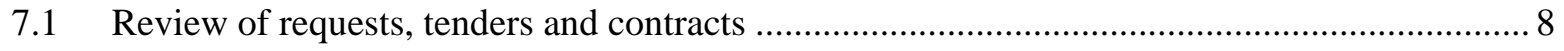

7.2 Selection, verification, and validation of methods ........................................................... 8

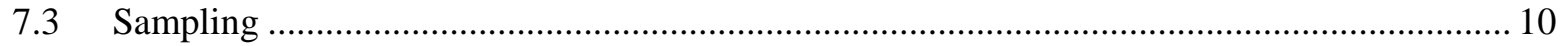



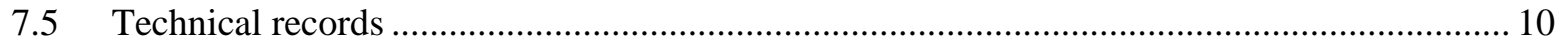

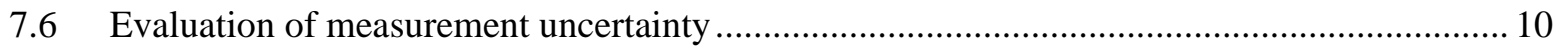

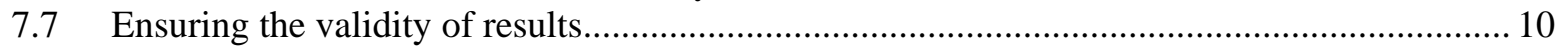

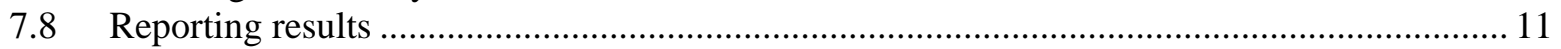

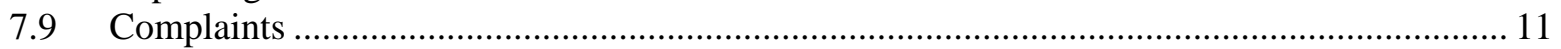

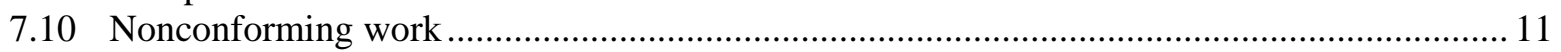

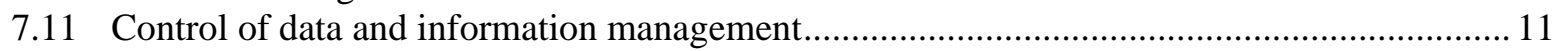

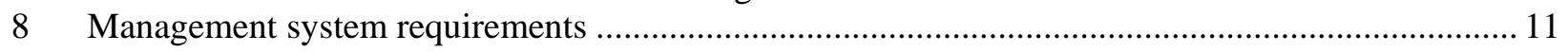

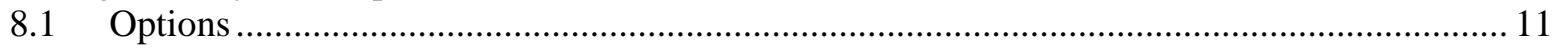

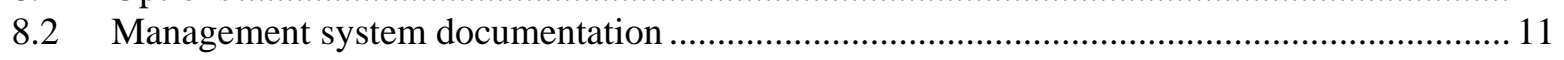

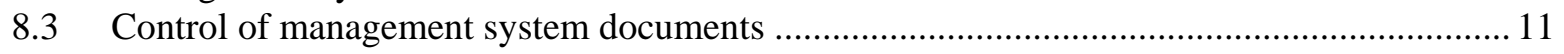

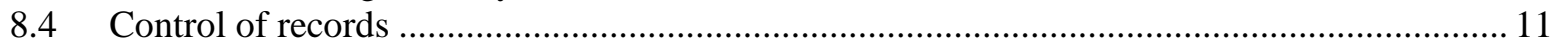

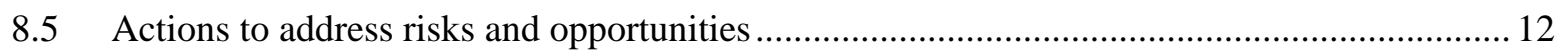

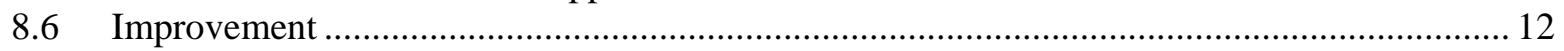




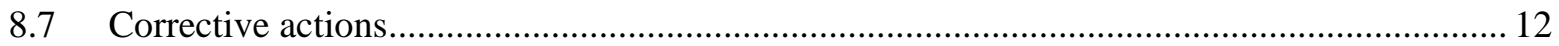

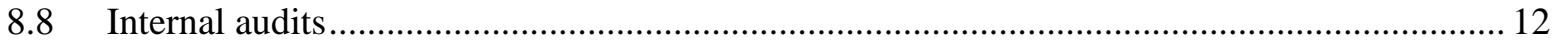

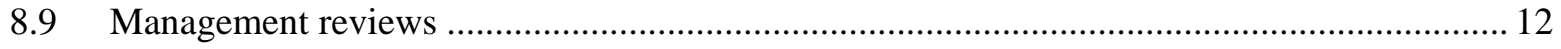

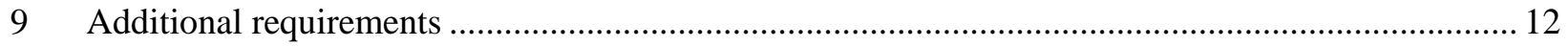




\section{General information}

\subsection{Scope}

1.1.1 NIST Handbook 150-8 specifies technical requirements and provides guidance for NVLAP accreditation of laboratories that provide acoustical testing services. This handbook supplements the NVLAP procedures and general requirements found in NIST Handbook 150, NVLAP Procedures and General Requirements.

1.1.2 This handbook, together with ISO/IEC 17025, NIST Handbook 150, NVLAP General Criteria Checklist, and NIST Handbook 150-8 Checklist constitute the collective body of requirements that must be met by a laboratory seeking NVLAP accreditation for acoustical testing services.

1.1.3 This handbook is intended for information and use by accredited acoustical testing services laboratories, assessors conducting on-site assessments, laboratories seeking accreditation, other laboratory accreditation systems, users of laboratory services, and others needing information on the requirements for NVLAP accreditation under the Acoustical Testing Services (ACO) Laboratory Accreditation Program (LAP).

\subsection{Organization of handbook}

The numbering and titles of the first three clauses of this handbook match those of NIST Handbook 150. However, unless there is an additional requirement, only the top-level numbering (e.g., 1, 2, and 3) is listed.

The numbering and titles of clauses four through eight of this handbook mirror those of ISO/IEC 17025. For clarity, the top-level and first sub-level clauses (e.g., 4, 5, 4.1, 4.2, etc.) are also numbered and titled to correspond with ISO/IEC 17025, even when there are no additional requirements given in this handbook.

\subsection{Program description}

1.3.1 This accreditation program is designed to satisfy the requirements of contractors, state and local governments, and federal agencies specifying accreditation for laboratories that perform testing for compliance to acoustical design specifications, performance testing of acoustical materials, noise producing devices, and noise protection devices.

1.3.2 Accreditation is available for standard test methods in the areas of sound absorption, acoustic impedance, sound attenuation, vibration, emitted sound power, sound pressure levels, and hearing protection, among others.

1.3.3 Test methods for which a laboratory may seek accreditation can be viewed on the NVLAP web site under the menu selection Search Test Methods or Calibration Parameters with the Acoustical Testing Service LAP selected as Program. Other test methods may be added to the program on request, if they are found to be appropriate by NVLAP. 


\subsection{References}

The following documents are referenced in this handbook. For dated references, only the edition cited applies. For undated references, the latest edition of the referenced document (including any amendments) shall apply within one year of publication or within another time limit specified by regulations or other requirement documents.

- NIST Handbook 150, NVLAP Procedures and General Requirements

- ASTM C634, Standard Terminology Relating to Environmental Acoustics

- ANSI S1.1, Acoustical Terminology

\subsection{Terms and definitions}

For the purposes of this handbook, the terms and definitions given in NIST Handbook 150, the terms and definitions given in the standards for which the laboratory seeks accreditation, and the terms and definitions given in ASTM C634 and ANSI S1.1 apply.

\subsection{Program documentation}

\subsubsection{General}

NVLAP assessors use NVLAP checklists to ensure that each laboratory receives an assessment comparable to that received by others. Checklists assist assessors in documenting the assessment to the NVLAP requirements found in ISO/IEC 17025, NIST Handbook 150 and program specific handbooks. Checklists contain definitive statements or questions about all aspects of the NVLAP criteria for accreditation, and form part of the On-Site Assessment Report (see NIST Handbook 150).

\subsubsection{NVLAP General Criteria Checklist}

All NVLAP programs use the NVLP General Criteria Checklist (formerly called the NIST Handbook 150 Checklist), which contains the requirements published in ISO/IEC 17025 and NIST Handbook 150. The checklist items are numbered to correspond to clauses 4 through 8 of ISO/IEC 17025:2017, and annexes A, B, and E of NIST Handbook 150. This checklist is available to applicant or accredited laboratories by request from NVLAP program managers after proof of ownership of a legal copy of the text of ISO/IEC 17025:2017 has been provided.

\subsubsection{NIST Handbook 150-8 Checklist}

The NIST Handbook 150-8 Checklist (also referred to as the ACO Program-Specific Checklist) addresses the requirements specific to the Acoustical Testing Services LAP. The checklist items are numbered to correspond to clauses 4 through 8 of ISO/IEC 17025. The current version of the Handbook 150-8 Checklist is available from the NVLAP web site at http://www.nist.gov/nvlap.

\subsubsection{Test Method Review Summary}

The assessor uses the Test Method Review Summary to review the laboratory's ability to perform the standard test methods for the Acoustical Testing Services LAP. The review of the test methods by the 
assessor ranges from observing tests to having laboratory staff describe the test procedures. The assessor notes on the Test Method Review Summary the depth into which each part of the test method was reviewed (Observed Test, Examined Apparatus, Walked/Talked Through Test, Listened to Description of Procedures).

\subsubsection{NVLAP Lab Bulletins}

NVLAP Lab Bulletins are issued to laboratories and assessors when needed to clarify program-specific requirements and to provide information about program additions and changes.

\section{$2 \quad$ LAP establishment, development and implementation}

This clause contains no information additional to that provided in NIST Handbook 150, clause 2.

\section{$3 \quad$ Accreditation process}

\subsection{General}

3.1.1 This clause discusses the assessment and accreditation process for laboratories in the Acoustical Testing Services LAP.

3.1.2 An overview of the laboratory accreditation process is provided in NIST Handbook 150, clause 3, and includes information pertaining to application for accreditation; on-site assessment; proficiency testing; accreditation decision; granting accreditation; renewal of accreditation; changes to scope of accreditation; monitoring visits; and suspension, denial, revocation, and voluntary termination of accreditation.

3.1.3 The assessment process consists of a NVLAP review of the laboratory's application and management system documentation and an on-site assessment visit.

3.1.4 NVLAP management may consider a pre-assessment on-site visit to better define a laboratory's requested scope of accreditation. In such cases, the pre-assessment costs will be charged to the laboratory in addition to the actual On-Site Assessment Fee.

\subsection{Management system review}

3.2.1 Prior to applying to NVLAP for accreditation, a laboratory should have a fully implemented management system. If the management system of the laboratory uses different numbering than that of ISO/IEC 17025, the laboratory shall create a cross-reference document allowing the laboratory and a NVLAP assessor to verify that all requirements of clauses 4 through 8 of the international standard, as well as other applicable general NVLAP requirements, are met by the management system. Additionally, the locations within the management system which the requirements given in this document, NIST Handbook 150-8, shall be provided in a cross-reference document. Note that the NVLAP General Criteria Checklist contains a column for the location of specific requirements within a laboratory's management system and, when completed, may serve as a core part of a cross-reference document. The checklist associated with this handbook may be similarly used. 
3.2.2 Prior to the on-site assessment, the NVLAP assessor will review laboratory documents to ensure they cover all aspects of the management system and, if followed, satisfy the requirements in ISO/IEC 17025, NIST Handbook 150, this handbook, and applicable test methods for which the laboratory seeks accreditation. The NVLAP assessor may also request technical documents, such as proficiency testing results, for review prior to arriving on-site. During the review, the NVLAP assessor may identify nonconformities and require changes to the management system so that it meets the requirements.

\subsection{On-site assessment}

3.3.1 When the management system review has been completed and identified nonconformities have been resolved, NVLAP schedules the on-site assessment.

3.3.2 The on-site assessment will take place at the laboratory site. The NVLAP assessor typically conducts the on-site assessment over a two- to three-day time period, where the length depends on the number of standard test methods for which a laboratory requests accreditation. The on-site assessment time may be longer if a laboratory is accredited for standard field-testing methods. The laboratory will be charged for extended on-site assessment time. Efforts will be made to minimize disruption to the normal working routines during the assessment. The NVLAP assessor will need time and workspace to complete assessment documentation during his/her time at the laboratory site.

3.3.3 The laboratory should have its facilities and equipment in good working order and be ready for examination according to the requirements identified in this handbook, ISO/IEC 17025, NIST Handbook 150, and the laboratory’s management system documents.

3.3.4 At the beginning of the on-site assessment, the laboratory should make available all supporting technical information in a format that is conducive to a detailed review.

3.3.5 NVLAP assessors will use the NVLAP General Criteria Checklist, the NIST Handbook 150-8 Checklist, and the Test Method Review Summary to record the results of the assessment. The checklists and the technical specifics contained in this handbook ensure that the assessment is complete and that all assessors cover the same items at each laboratory.

3.3.6 The activities covered during a typical on-site assessment are discussed below. The NVLAP assessor, prior to the visit, will provide a preliminary agenda, which may change due to findings observed during the on-site assessment.

a) Opening meeting: The NVLAP assessor will meet with laboratory management, supervisory personnel, and other staff members at the discretion of laboratory's management to explain the purpose of the on-site assessment and to discuss the schedule for the assessment activities. Information provided by the laboratory in its application may be discussed during this meeting.

b) Staff interviews: The assessor will ask the laboratory manager to assist in arranging times for individual interviews with laboratory staff members. The assessor will interview staff members filling key positions (e.g., Laboratory Manager, Quality Manager, Authorized Representative) and other staff members who have an effect on the outcome of the testing. The assessor does not need to talk to all staff members; however, the assessor will select staff members representing all aspects of the laboratory. These interviews are conducted to determine if the staff members are properly trained, assigned, and supervised; are technically competent for the tasks assigned to 
them; and are implementing their assigned aspects of the quality management in compliance with it.

c) Records review: The assessor will review laboratory documentation, including the management system, quality policies and procedures, equipment and maintenance records, record-keeping procedures, testing procedures, laboratory test records and reports, personnel competency records, personnel training plans and records, and safeguards for the protection of sensitive and proprietary information. The assessor may request additional information in an effort to clarify issues regarding a nonconformity or to delve more deeply into a technical issue. Laboratory staff should be available to answer questions.

d) Internal audit and management review: The assessor will review and discuss with the laboratory staff the laboratory's internal audit and management review activities. The discussion will include all aspects of those activities including the management system procedures, the audit findings, the results of the management review, and the actions taken to resolve those findings.

e) Equipment and software: The assessor will examine and determine the suitability of all equipment and facilities required to perform the standard test methods for which the laboratory is accredited (or is seeking accreditation). The appropriate environmental conditions required for testing will be assessed. The assessor will review acoustical test room qualifications and test data, examine hardware and software for function and appropriateness, and review software validation and verification procedures.

f) Demonstrations: Based on the scope of accreditation, the assessor will observe demonstrations of selected testing procedures conducted by technical personnel assigned to conduct the tests and will discuss the tests to assure that the staff understands the procedures. The assessor may select and trace the history of one or more samples from receipt to final issuance of the test reports.

g) Proficiency testing: The assessor will discuss all aspects of proficiency testing results with appropriate staff. Test methodology and records documenting the laboratory's execution of the testing will be reviewed and discussed. Unusual trends and outlying results will be discussed.

h) On-site assessment report: The assessor will complete an on-site assessment report, which summarizes the findings and clearly lists nonconformities and comments (positive or negative). This report normally consists of the Narrative Summary, the NVLAP General Criteria Checklist, the NIST Handbook 150-8 Checklist, and the Test Method Review Summary.

i) Closing meeting: The assessor will conduct a closing meeting with the laboratory manager, supervisory personnel, the authorized representatives, and other staff members at the discretion of the laboratory's management to discuss the findings. During the visit, the assessor will have categorized each issue identified as either a nonconformity or a comment; these will then be discussed at the closing meeting. The assessor will specifically note items that have been corrected during the on-site assessment along with any requirements for additional action.

The process for resolving nonconformities identified during the on-site assessment is documented in Section 3.3.4 of NIST Handbook 150. Any unresolved disagreements between the laboratory and the assessor may be referred to NVLAP headquarters for resolution. All information obtained by the assessor is held in strictest confidence.

The first page of the on-site assessment report is signed by the assessor and the laboratory Authorized Representative to acknowledge the discussion, but this does not necessarily indicate 
agreement by the laboratory. A copy of the report is given to the laboratory representative for retention, and the assessor sends the original to NVLAP.

3.3.7 The laboratory's response to all nonconformities should be clearly documented, providing a reference to applicable sections in the management system, the assessor's on-site assessment report, and any other technical supporting information. If found to be incomplete, NVLAP may reject the laboratory's response submission and request that the laboratory resubmit and provide the necessary documentation in order to facilitate a complete review of the resolved nonconformities.

3.3.8 The laboratory should review all comments for potential improvements in acoustical testing. It is not required that actions be taken in response to comments; nor is there a requirement to notify NVLAP of any response or non-response to comments.

\subsection{Proficiency testing}

3.4.1 The laboratory shall have a plan for participating in proficiency testing (e.g., interlaboratory comparisons).

3.4.2 Laboratories shall participate in interlaboratory comparisons (ILCs) sponsored by standards organizations (ASTM International, ANSI, etc.) when the laboratory is accredited for the test method that is being used in the ILC.

3.4.3 Laboratories shall participate in proficiency testing activities announced by NVLAP.

3.4.4 Procedures for receiving, analyzing, and monitoring the laboratory's proficiency test results shall be part of the laboratory's quality system documentation.

3.4.5 The laboratory shall evaluate the proficiency testing results, identify all outliers, and follow the requirements of its process for the control of nonconforming work.

3.4.6 The laboratory shall correct the issues(s) that led to the unsatisfactory performance in proficiency testing.

\section{General requirements}

\subsection{Impartiality}

There are no requirements additional to those set forth in ISO/IEC 17025.

\subsection{Confidentiality}

There are no requirements additional to those set forth in ISO/IEC 17025. 


\section{Structural requirements}

There are no requirements additional to those set forth in ISO/IEC 17025.

\section{Resource requirements}

\subsection{General}

There are no requirements additional to those set forth in ISO/IEC 17025.

\subsection{Personnel}

6.2.2.1 The laboratory shall maintain records of personnel designated to fulfill NVLAP requirements including: Laboratory Director, Quality Manager, NVLAP Authorized Representative, and NVLAP Approved Signatories.

NOTE The staff information may be kept in the official personnel folders or in separate, official folders that contain only information that the NVLAP assessors need to review. The assessors do not need to see any documents not related to the accreditation of the laboratory.

6.2.3.1 The laboratory shall establish and document performance criteria used to determine that a staff member is qualified to work independently.

6.2.3.2 Staff members shall be retrained when procedures change, laboratory equipment and/or test chambers change, scope of accreditation changes, or when the individuals are assigned new responsibilities. Each staff member may receive training for assigned duties either through on-the-job training, formal classroom study, attendance at conferences, or another appropriate mechanism.

6.2.3.3 Training materials that are maintained within the laboratory shall be kept up-to-date and readily available to laboratory staff.

6.2.3.4 For each staff member, the staff member's immediate supervisor, or a designee appointed by the Laboratory Director, shall annually conduct and document an assessment and an observation of performance.

\subsection{Facilities and environmental conditions}

There are no requirements additional to those set forth in ISO/IEC 17025.

\subsection{Equipment}

There are no requirements additional to those set forth in ISO/IEC 17025. 


\subsection{Metrological traceability}

6.5.1.1 Proper performance of calibrated testing equipment shall be periodically verified under routine use and when the test equipment has been shipped or transported.

6.5.1.2 The performance verification shall be documented in the laboratory calibration/verification $\log (\mathrm{s})$ or other suitable record.

6.5.1.3 The laboratory shall determine equipment calibration intervals based on the frequency of use of the equipment and the environment in which it is used, and/or in accordance with standard test methods.

6.5.1.4 The laboratory shall document that the calibration intervals used by the laboratory are sufficient.

6.5.1.5 The reference standards used and the environmental conditions at the time of calibration shall be documented for all calibrations.

6.5.1.6 A laboratory may calibrate its own measurement and test equipment if it has trained personnel, written instructions, and the proper equipment and environment to provide traceability.

6.5.1.7 Calibration records and evidence of the traceability of the reference standards used shall be made available for inspection during the on-site visit.

\subsection{Externally provided products and services}

There are no requirements additional to those set forth in ISO/IEC 17025.

\section{$7 \quad$ Process requirements}

\subsection{Review of requests, tenders and contracts}

There are no requirements additional to those set forth in ISO/IEC 17025.

\subsection{Selection, verification, and validation of methods}

7.2.1.1 The laboratory may be accredited to perform standard test methods in their entirety or to perform only certain sections in the test method.

7.2.1.2.1 The laboratory shall follow the current version of the standard test method for laboratory methods and field testing methods.

7.2.1.2.2 When a standard test method is revised, the laboratory shall implement the new version within 6 months.

7.2.1.2.3 The most recent editions of the documents listed in section 1.4 shall be available as references in maintaining the management system. 
7.2.1.3.1 The laboratory shall have written procedures for laboratory personnel to follow when conducting tests. The procedures may reference (versus restating) the procedure in a standard method. Further, the laboratory's written procedures:

a) shall address any information not specifically contained in the standard method and any deviations used by the laboratory, and

b) shall include equipment operation, calibration checks, and quality control checks.

7.2.1.3.2 If a regulation requires testing against previous versions of a standard test method, then the laboratory:

a) shall document that requirement and

b) shall have available the required version of the standard test method.

7.2.1.3.3 Except as indicated in 7.2.1.3.2 above, when customers request testing to previous or obsolete versions of a standard test method, the laboratory shall clearly identify to the customer that the testing is not part of their current scope of accreditation.

\subsubsection{Standard field-testing methods}

7.2.1.8.1 A laboratory may be accredited for standard field-testing methods.

7.2.1.8.2 The field test may be performed in the laboratory only, or in both the laboratory and field, or in the field only.

7.2.1.8.3 If a laboratory selects standard field-testing methods to be included in its scope of accreditation, the laboratory shall provide to the NVLAP assessor the following:

a) complete step-by-step procedure for personnel to follow when performing the standard field test;

b) demonstration or mock-up of the test procedure;

c) folder or file containing raw data for a specific standard field test previously performed by the laboratory;

d) test reports and test data sheets.

7.2.1.8.4 The laboratory shall select an appropriate acoustic component (e.g., office wall partition) within its facility as the designated field testing reference specimen.

7.2.1.8.5 All quality control and monitoring procedures outlined both in ISO/IEC 17025 and in this handbook shall apply to the field testing reference specimen.

\subsubsection{Parallel standard test methods}

7.2.1.9.1 Some standard test methods produced by the American National Standards Institute (ANSI), the International Organization for Standardization (ISO), and other standards organizations may be sufficiently alike to allow for parallel accreditation.

7.2.1.9.2 A laboratory may request that its scope of accreditation reflect parallel standard test methods. 
7.2.1.9.3 The laboratory shall provide evidence that the laboratory meets the requirements of the parallel standard test method.

7.2.1.9.4 NVLAP will determine if the laboratory meets all the requirements before adding the parallel standard test method to the laboratory's scope of accreditation.

\subsection{Sampling}

There are no requirements additional to those set forth in ISO/IEC 17025.

\subsection{Handling of test or calibration items}

There are no requirements additional to those set forth in ISO/IEC 17025.

\subsection{Technical records}

7.5.1.1 Records shall be maintained for at least three years.

7.5.1.2 All technical records (test/calibration/verification, etc.), in both hard-copy and electronic format, shall include the identity of the personnel responsible for the preparation, calibration, testing, and checking of the results and, where appropriate, the associated document date.

\subsection{Evaluation of measurement uncertainty}

There are no requirements additional to those set forth in ISO/IEC 17025.

\subsection{Ensuring the validity of results}

7.7.1.1 The laboratory shall choose an appropriate reference specimen(s) to be used when conducting tests.

7.7.1.2 The reference specimen(s) shall be tested annually and whenever a change is made to the test chambers or test instrumentation.

7.7.1.3 The analysis of test data for the purpose of quality control shall be presented in a suitable format, such as a table and/or a graph, for review and interpretation by staff and the NVLAP assessor. The quality control data may be presented by hard copy or electronically.

7.7.1.4 The laboratory should experimentally characterize the sound field(s) by measuring and documenting sound pressure at a number of different locations and, from these measurements, obtain an estimate of the mean and standard deviation of the sound field when determining, e.g., sound absorption, sound power output, transmission loss, etc.

7.7.1.5 When computer software is purchased, updated, and/or algorithms revised, the laboratory shall manually verify, through use of a standard data set, the accuracy of output results before using the computer software for reporting valid tests. 


\subsection{Reporting results}

7.8.3.3 Test report templates shall be developed for each standard test method for which the laboratory requests accreditation.

7.8.3.4 Test reports shall provide all necessary information to permit the same or another laboratory to reproduce the test plan.

7.8.3.5 When a test report contains results from tests that are outside the laboratory's scope of accreditation (e.g., testing is performed against previous or obsolete versions of a standard test method), the results shall be clearly identified.

7.8.3.6 The measurement uncertainty shall be reported numerically in relation to the test results if required by the test method or the customer.

\subsection{Complaints}

There are no requirements additional to those set forth in ISO/IEC 17025.

\subsection{Nonconforming work}

There are no requirements additional to those set forth in ISO/IEC 17025.

\subsection{Control of data and information management}

There are no requirements additional to those set forth in ISO/IEC 17025.

\section{$8 \quad$ Management system requirements}

\subsection{Options}

There are no requirements additional to those set forth in ISO/IEC 17025.

\subsection{Management system documentation}

There are no requirements additional to those set forth in ISO/IEC 17025.

\subsection{Control of management system documents}

There are no requirements additional to those set forth in ISO/IEC 17025.

\subsection{Control of records}


There are no requirements additional to those set forth in ISO/IEC 17025.

\subsection{Actions to address risks and opportunities}

There are no requirements additional to those set forth in ISO/IEC 17025.

\subsection{Improvement}

There are no requirements additional to those set forth in ISO/IEC 17025.

\subsection{Corrective actions}

There are no requirements additional to those set forth in ISO/IEC 17025.

\subsection{Internal audits}

8.8.1.1 The internal audit shall cover compliance with ISO/IEC 17025, NVLAP, laboratory management system, regulatory, test standard, and contractual requirements.

8.8.1.2 A laboratory applying to NVLAP for the first time shall conduct at least one complete internal audit and submit the audit records to NVLAP with the management system documentation prior to the first on-site assessment.

8.8.1.3 For accredited laboratories, records of internal audits conducted since the previous on-site assessment shall be available for review during an on-site assessment.

\subsection{Management reviews}

8.9.1.1 A laboratory applying to NVLAP for the first time shall conduct at least one complete management review and submit the records to NVLAP with the management system documentation prior to the first on-site assessment.

8.9.1.2 For accredited laboratories, records of management reviews conducted since the previous on-site assessment shall be available for review during an on-site assessment.

\section{$9 \quad$ Additional requirements}

There are no additional requirements beyond ISO/IEC 17025, NIST Handbook 150 and its associated normative annexes, and other normative references cited in this handbook. 